\title{
Task-Induced Modulation of Intrinsic Functional Connectivity Networks in the Behaving Rat
}

\author{
Jennifer Li, ${ }^{1}$ Sarah Martin, ${ }^{1}$ Mark D. Tricklebank, ${ }^{1}{ }^{\oplus}$ Adam J. Schwarz, ${ }^{2,3}$ and Gary Gilmour ${ }^{1}$ \\ ${ }^{1}$ Centre for Cognitive Neuroscience, Eli Lilly and Company Limited, Windlesham, Surrey GU20 6PH, United Kingdom, ${ }^{2}$ Tailored Therapeutics, Eli Lilly and \\ Company, Indianapolis, Indiana 46285, ${ }^{3}$ Department of Psychological and Brain Sciences, Indiana University, Bloomington, Indiana 46285
}

While resting-state functional magnetic resonance imaging can probe intrinsic network connectivity in both human and rodent brain, behavioral modulation of these connectivity patterns has not yet been demonstrated in the rodent due to the requirements of immobilization or anesthesia for MRI scanning. To enable the effects of behavioral tasks on functional connectivity to be measured in freely moving, awake rats, implanted carbon paste electrodes (CPEs) were used to monitor low-frequency fluctuations of tissue oxygenation. Rats were implanted with CPEs in two nodes of the default mode network (DMN) and two nodes in a lateral cortical network, revealing amperometric oxygen correlation patterns consistent with imaging studies. Using a block design study where rats alternated between sustained periods of instrumental response and unscheduled spontaneous behavior, task-induced decreases in functional connectivity were observed between the DMN node pair, but not in the distinct lateral cortical network, demonstrating network-specific modulation of functional connectivity.

Key words: default mode network; functional connectivity; oxygen amperometry

\section{Introduction}

Correlated low-frequency oscillations in the blood oxygen $\left(\mathrm{O}_{2}\right)$ level-dependent (BOLD) resting-state functional magnetic resonance imaging (rsfMRI) signal are now an established means of assessing intrinsic connectivity of functional networks in the human brain (Beckmann et al., 2005; Fox et al., 2005; Smith et al., 2009). These signals have been shown to be a sensitive measure of pathological states in the task-free ("resting") state (for review, see Fox and Greicius, 2010). Furthermore, researchers have obtained a deeper understanding of how intrinsic network activity relates to behavioral states by combining assessments of functional connectivity with specific cognitive tasks (Esslinger et al., 2009; Shirer et al., 2012). Recently, distinct task-free intrinsic connectivity networks have also been demonstrated in rodents (Becerra et al., 2011; Sforazzini et al., 2014), providing a potentially powerful translational probe of brain function. However, since rodent rsfMRI studies are performed in an anesthetized or immobilized state, the ability to investigate functional connectivity during active behavior is precluded or greatly limited. The degree to which functional connectivity in the rodent can be modulated by specific cognitive or behavioral states remains unknown. In vivo $\mathrm{O}_{2}$ amperometry is an alternative to rodent fMRI that can measure real-time changes in extracellular $\mathrm{O}_{2}$ via in-

Received Aug. 20, 2014; revised Nov. 5, 2014; accepted Nov. 16, 2014.

Author contributions: J.L., M.D.T., A.J.S., and G.G. designed research; J.L. and S.M. performed research; J.L., S.M., and G.G. analyzed data; J.L., M.D.T., A.J.S., and G.G. wrote the paper.

We thank John Huxter for providing guidance and assistance with the functional connectivity analysis.

All authors are employees of Eli Lilly. The authors declare no competing financial interests.

Correspondence should be addressed to Jennifer Li, DPhil, Lilly Centre for Cognitive Neuroscience, Eli Lilly \& Co. Ltd, Erl Wood Manor, Windlesham, Surrey GU20 6PH, UK. E-mail: lije@lilly.com.

DOI:10.1523/JNEUROSCI.3488-14.2015

Copyright $\odot 2015$ the authors $\quad 0270-6474 / 15 / 350658-08 \$ 15.00 / 0$ dwelling probes in freely moving animals. This technique has been successfully used to measure regional brain tissue $\mathrm{O}_{2}$ levels with subsecond temporal resolution in a variety of behavioral paradigms (Francois et al., 2012; McHugh et al., 2013), and more recently to demonstrate that inter-region low-frequency correlations of the $\mathrm{O}_{2}$ signal can be pharmacologically modulated ( $\mathrm{Li}$ et al., 2014).

To extend the cross-species translation of functional connectivity measures beyond the "resting state," $\mathrm{O}_{2}$ amperometry was used in the present work to assess relationships within and between two distinct brain networks in freely moving rats engaged in periods of instrumental responding for food reward. The foci were the default mode network (DMN), for which a rodent homolog has recently been identified (Lu et al., 2012; Schwarz et al., 2013a; Sforazzini et al., 2014), and a lateral cortical network (LCN) extending from the frontal eye fields in anterior secondary motor cortex through sensorimotor regions to insula cortex. LCN has been shown to be uncorrelated with the DMN in rsfMRI studies (Schwarz et al., 2013b; Sforazzini et al., 2014).

\section{Materials and Methods}

Subjects. All experiments were conducted in accordance with the Animals (Scientific Procedures) Act 1986 and local ethical requirements. Thirtysix male Wistar rats (284-318 g at surgery) were surgically prepared at Charles River and delivered to Eli Lilly, where they were group-housed in cages of four in a temperature-controlled $\left(20-22^{\circ} \mathrm{C}\right)$ environment with food and water available ad libitum under a $12 \mathrm{~h}$ light/dark cycle.

Surgery. Under isoflurane anesthesia, animals were implanted unilaterally with four carbon paste electrodes (CPEs) in medial prefrontal cortex, prelimbic region [PRL; anteroposterior (AP), + $4.0 \mathrm{~mm}$; mediolateral (ML), $-0.4 \mathrm{~mm}$; dorsoventral (DV), $-2.2 \mathrm{~mm}$ from dura]; anterior secondary motor cortex $(\mathrm{M} 2$; $\mathrm{AP},+4.0 \mathrm{~mm}$; $\mathrm{ML},-3.2 \mathrm{~mm}$; DV, $-1.2 \mathrm{~mm}$ from dura); primary somatosensory cortex, jaw region (S1); 


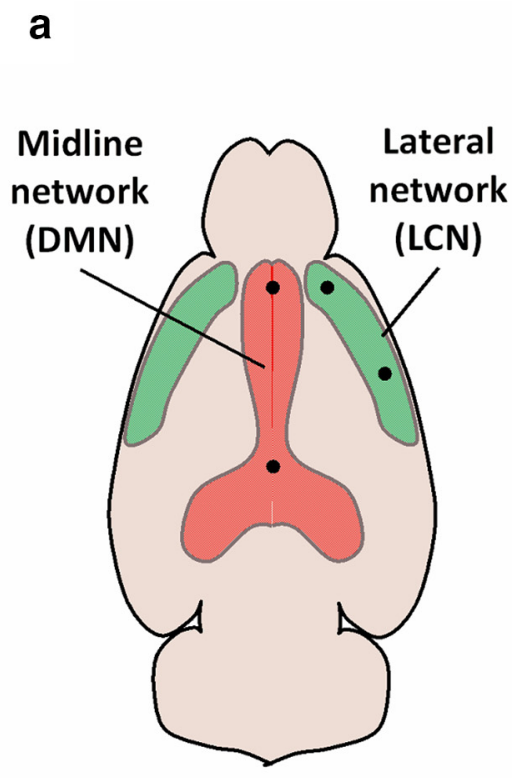

b
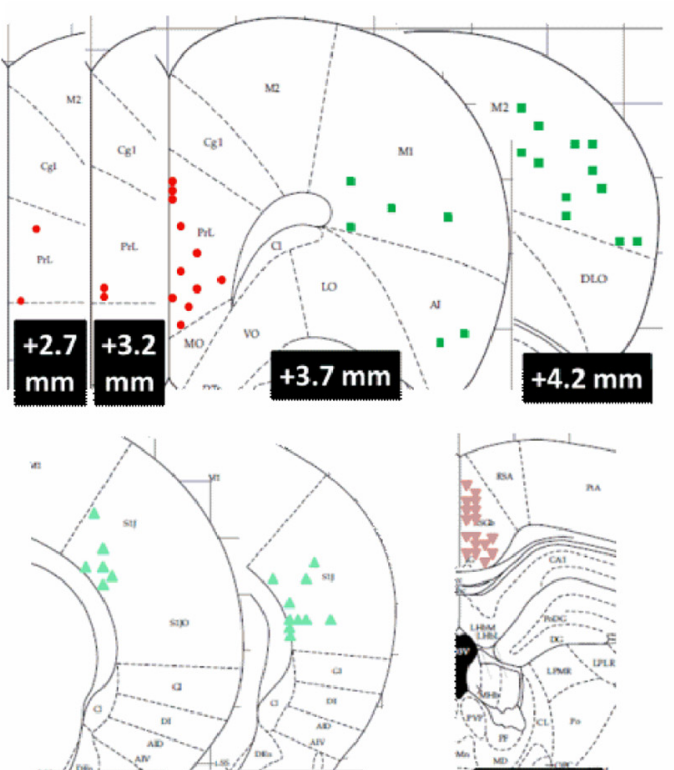

$+1.6 \mathrm{~mm}+1.7 \mathrm{~mm}$

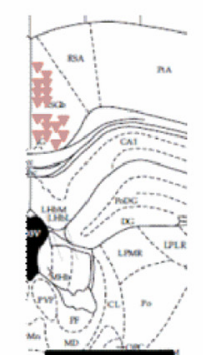

$-3.8 \mathrm{~mm}$

C
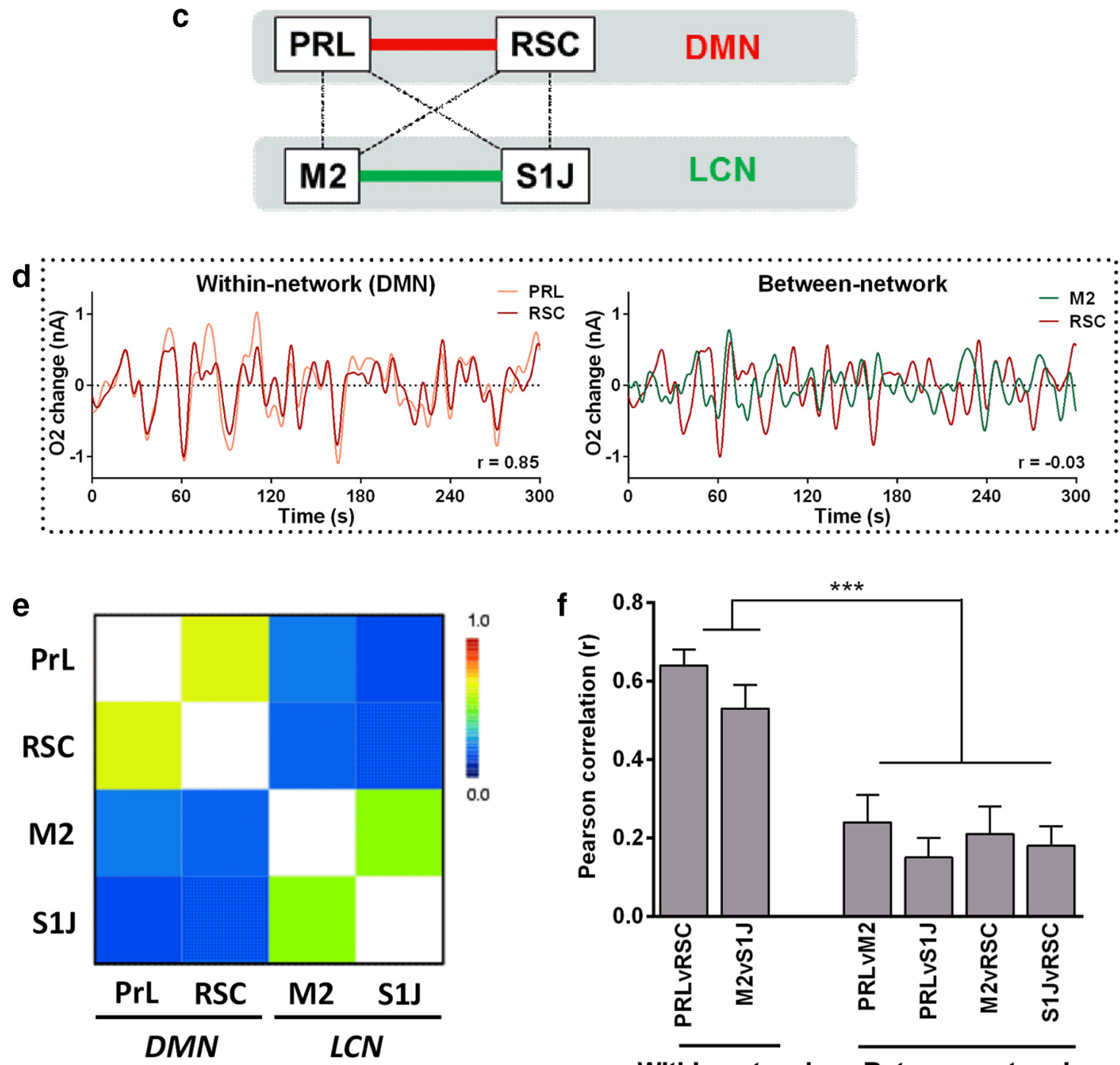

Within-network $\overline{\text { Between-network }}$ 
$\mathrm{AP},+1.6 \mathrm{~mm}$; ML, $-5.0 \mathrm{~mm}$; DV , $-1.8 \mathrm{~mm}$ from dura); and the retrosplenial cortex (RSC; AP, $-4.0 \mathrm{~mm}$; ML, $-0.4 \mathrm{~mm}$; DV, $-1.5 \mathrm{~mm}$ from dura). The reference electrode was placed in posterior cortex and the auxiliary electrode was wrapped around one posterior skull screw. Preoperative and postoperative Rimadyl (carprofen, $5 \mathrm{mg} / \mathrm{kg}$, s.c.; Pfizer) was administered, and animals were allowed to recover in thermostatically controlled cages. A postoperative recovery period of 2 weeks was allowed before testing commenced. At the start of testing, animals weighed $344-510 \mathrm{~g}$. Animals were food restricted to $\sim 2.5 \mathrm{~g}$ of chow per rat daily for $7 \mathrm{~d}$ before testing began.

In vivo amperometry. Changes in extracellular tissue $\mathrm{O}_{2}$ were measured using constant potential amperometry with CPEs (Lowry et al., 1997). A potential of $-650 \mathrm{mV}$ was applied to electrodes, allowing the electrochemical reduction of dissolved $\mathrm{O}_{2}$ at their tip (Bolger et al., 2011). CPEs were constructed from $8 \mathrm{~T}$ (200 $\mu \mathrm{m}$ bare diameter, $270 \mu \mathrm{m}$ coated diameter) Teflon-coated silver wire (Advent Research Materials). The Teflon insulation was slid along the wire to create a 2-mm-deep cavity, which was packed with carbon paste (prepared according to O'Neill et al., 1982). Before implantation, all CPEs were calibrated in vitro in a glass cell containing $15 \mathrm{ml}$ of phosphate buffer solution $(0.01 \mathrm{M}), \mathrm{pH} 7.4$, saturated with nitrogen $\left(\mathrm{N}_{2}\right)$ gas, atmospheric air (from a Rena air pump), or pure $\mathrm{O}_{2}$ (compressed gas) at room temperature. The concentrations of dissolved $\mathrm{O}_{2}$ were taken as $0 \mu \mathrm{M}\left(\mathrm{N}_{2}\right.$-saturated), $240 \mu \mathrm{M}$ (air-saturated), and $1260 \mu \mathrm{M}\left(\mathrm{O}_{2}\right.$-saturated $)$, respectively. Reference and auxiliary electrodes were also prepared from $8 \mathrm{~T}$ Teflon-coated silver wire by removing $2 \mathrm{~mm}$ of Teflon from the tip. All electrodes were soldered to gold connectors, which were cemented into six-pin plastic sockets (both from Plastics One) during surgery.

Equipment and data recording. Animals were tested in operant test chambers (ENV 008, Med Associates), constructed of two aluminum walls and two Perspex walls. The floor consisted of 19 stainless steel bars (diameter, $0.48 \mathrm{~cm}$; spaced $1.6 \mathrm{~cm}$ apart) and each chamber had a house light (100 mA; ENV 215M, Med Associates), located $1.8 \mathrm{~cm}$ from the ceiling on the opposite wall to a food magazine incorporating a magazine light, and pellet dispenser for reward (sucrose pellet) delivery. Infrared beam breaks in the magazine recorded the number of nose pokes. Some sessions (days 4, 9, 12, 13, and 17 of acquisition and all extinction/reinstatement sessions) were recorded using a ceiling-mounted infrared video camera and recorded videos were analyzed by in-house software that calculated pixel changes per second.

During $\mathrm{O}_{2}$ signal recording, the head-mounted six-pin socket was connected to a low-noise, four-channel potentiostat (EA164 Quadstat, eDAQ) via a flexible screened six-core cable mounted through a swivel (both Plastics One) in the ceiling of the cage to allow free movement of the animal throughout the test chamber. A 16-channel e-corder (ED1621,eDAQ) was used for analog-to-digital conversion before data were collected on a PC running Chart v5 (both eDAQ). Amperometric recordings were recorded at $1000 \mathrm{~Hz}$ and analyzed at a down-sampled frequency of $200 \mathrm{~Hz}$.

In vivo calibration. Before the study began, the electrodes were calibrated in vivo to assess their responsiveness to the inhalation of different concentrations of $\mathrm{O}_{2}$ in freely moving rats. Animals were placed individually in test chambers and a constant $-650 \mathrm{mV}$ potential was applied to CPEs to allow signal settling for $15 \mathrm{~min}$. The rats were then exposed to $\mathrm{O}_{2}$ gas ( $\sim 200 \mathrm{psi})$ at their nose for $30 \mathrm{~s}$ and the $\mathrm{O}_{2}$ signals were allowed to

\section{$\leftarrow$}

Figure 1. Distinct intrinsic networks measured with $\mathrm{O}_{2}$ amperometry at rest. $\boldsymbol{a}, \mathrm{O}_{2}$ probes targeted two regions of the DMN (PRL and RSC) and two regions of the LCN (M2 and S1J). $\boldsymbol{b}$, Final placements of $\mathrm{O}_{2}$ probes based on histological analysis. c, The network connectivity pairs obtained. $\boldsymbol{d}$, Raw regional $\mathrm{O}_{2}$ signals filtered between 0.01 and $0.1 \mathrm{~Hz}$ from one representative animal over 5 min at rest. Left panel shows within-network regions of the DMN (PRL and RSC) with highly synchronous fluctuations. Right panel shows between-network regions (RSC and M2) with uncorrelated low-frequency fluctuations. $\boldsymbol{e}$, Correlation matrix of functional connectivity $(r)$ during unscheduled spontaneous behavior. $\boldsymbol{f}$, Bar chart of connectivity of each node pair, mean $r \pm$ SEM, $n=19$. Significant between-network versus within-network pair difference $\left({ }^{* *} p<0.0001\right)$.
}

decay back to baseline levels. This process was repeated three times with $\mathrm{O}_{2}$ and then replicated with $\mathrm{N}_{2}$ gas.

Testing schedule. Animals were placed in test chambers and a constant $-650 \mathrm{mV}$ potential was applied to CPEs to allow signal settling for 15 min before the recording session began on each day. A blocked design of four alternating periods of an instrumental responding task and rest (unscheduled spontaneous behavior) was used. During "task" blocks, animals were cued by sound (clicker $0.5 \mathrm{~s}$ before the start of each task block) and light (illumination of house and magazine light for the duration of the 15 min task block). The cues were signals to nose poke for food reward (sucrose pellet) under a variable-interval $30 \mathrm{~s}$ schedule (range, 7-53 s). During "rest" blocks, cues and rewards were removed and behavior was not schedule controlled. $\mathrm{O}_{2}$ signals (and videos on selected days) were recorded for the duration of the 60 min testing session. At the conclusion of each session, rats were returned to their home cages. Animals were trained on this schedule once daily until behavior was stably acquired $(18 \mathrm{~d})$. At this point, animals were exposed in a counterbalanced manner to three different control conditions: Extinction, All-Task, and All-Rest. Extinction involved withholding reward while the cues continued to be presented for 3 consecutive days. All-Task involved a single 60 min block of scheduled and cued behavior. All-Rest involved a single 60 min block of unscheduled spontaneous behavior. Each control condition was followed by $3 \mathrm{~d}$ of reinstatement, where the rewards were returned to the original block design, before being subjected to the next control condition.

Throughout test sessions, three behavioral measures were recorded and summarized for each 15 min block. These were the number of nose pokes, the number of pellets dispensed, and the level of movement monitored (indexed as a magnitude in pixel change).

Data analysis. For each electrode, linear interpolation was used postcollection to replace occasional missing data points and a biquad Butterworth filter (high-pass $0.1 \mathrm{~Hz}$ ) was used to suppress fast noise-related artifacts. Data were normalized by subtraction of the $60 \mathrm{~s}$ average presession value from each data point in the series, thereby compensating for absolute differences in baselines between channels. Finally, a boxcaraveraging algorithm was applied to down-sample the data, keeping a single average from multiple $0.5 \mathrm{~s}$ nonoverlapping windows. The time courses of absolute regional $\mathrm{O}_{2}$ levels were plotted in $2 \mathrm{~min}$ bins, and the area under curve (AUC) was calculated for each 15 min block on data normalized to the final $60 \mathrm{~s}$ of the previous block to show a relative amplitude response for each block. Functional connectivity data were analyzed in the $0.01-0.1 \mathrm{~Hz}$ range, from a period $600 \mathrm{~s}$ presession start to $3600 \mathrm{~s}$ postsession start (incorporating all four $15 \mathrm{~min}$ blocks), and averaged in $300 \mathrm{~s}$ time bins. To achieve this, the session start-aligned series of $N$ data points from both brain regions were padded with N/4 additional points to eliminate edge artifacts, which are produced when applying a low-pass filter to data with a mean offset from zero. Butterworth noise filtering was applied as before for each frequency $F$ of interest and then data were correlated in a series of half-overlapping windows of length $2 / F$, excluding padded sections of data. To build a correlation spectrum over time for multiple frequency bins, Pearson's $r$ was generated as an index of functional connectivity between the filtered signals at a given time for that frequency. An average $r$ value for each 15 min block was calculated for each pair of regions on each day, and the resting withinnetwork/between-network comparison and correlation matrix was shown as an average of both rest blocks.

Statistics. For behavioral measures, the 15 min block summary data from each day were analyzed in a repeated-measures ANOVA with time point (Task1/Rest1 or Task2/Rest2) and block state (task or rest) as the within-subjects factors. For functional connectivity analysis, broadband $(0.1-0.01 \mathrm{~Hz})$ correlation values (Pearson's $r$ ) summarized in $15 \mathrm{~min}$ blocks underwent Fisher's $z$-transformation before a repeated-measures ANOVA with network (DMN: PRL-RSC; or LCN: M2-S1J), time point (Task1/Rest1 or Task2/Rest2) and block state (task or rest) as withinsubjects factors was performed. Mauchly's sphericity test was performed on all correlation data and Greenhouse-Geisser adjustments applied to degrees of freedom as necessary. If a significant effect of block state or a significant network-block state interaction was seen, planned comparisons of the block state (task vs rest) within each network was performed. 
a

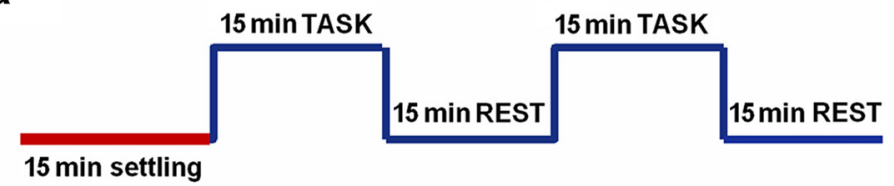

b

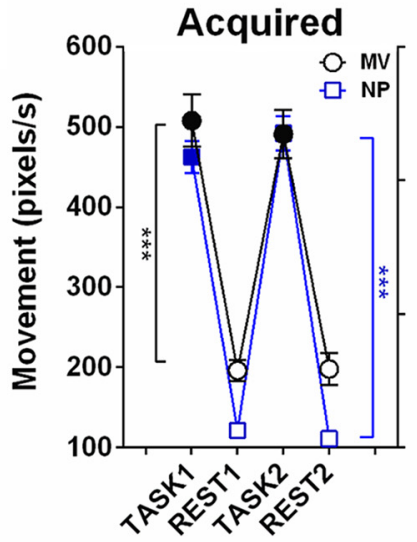

C

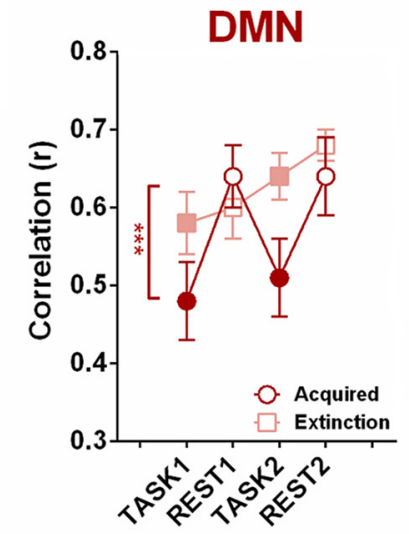

d
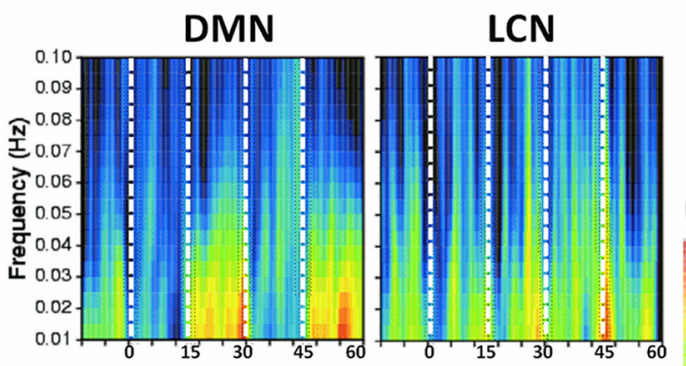

0.8

Acquired
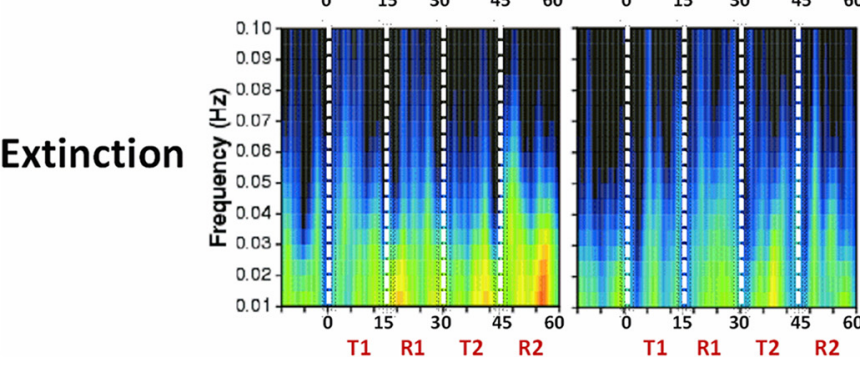

Figure 2. Task-induced modulation of behavioral state and functional connectivity. $\boldsymbol{a}, 15 \mathrm{~min}$ task/rest block design. $\boldsymbol{b}$, Behavioral measures (MV, movement; NP, nose pokes) per block during acquired and extinguished days. Both parameters show significant task versus rest differences (both $p<0.001$ ), mean \pm SEM $n=18-19$. c, Broadband correlations of DMN and LCN on acquired and extinguished days per block. Significant task versus rest differences were found only for acquired day DMN activity $(p<0.001)$, mean $r \pm$ SEM, $n=19$. $d$, Heat maps of DMN and LCN connectivity across $0.01-0.1 \mathrm{~Hz}$ frequencies over the session on acquired and extinguished days. Color represents $r$ values between 0.3 and $0.8, n=19$; time on the $x$-axis is in minutes.
For the within/between network comparison at rest, the combined rest $r$ values (average of Rest1 and Rest2) for each pair of regions underwent a repeated-measures ANOVA with region pair as the within-subjects factor. If significant, a planned comparison of the within-network pairs (PRL-RSC and M2-S1J) versus the between-network pairs (PRL-M2, PRL-S1J, M2-RSC, S1J-RSC) was performed. For absolute regional $\mathrm{O}_{2}$ levels, AUC values were analyzed by a repeated-measures ANOVA with region (PRL, RSC, M2, S1J), time point (Task1/Rest1 or Task2/Rest2), and block state (task or rest) as within-subjects factors. If a significant effect of block state or a significant region-block state interaction was seen, planned comparisons of the block state (task vs rest) for regions within each network (DMN: PRL and RSC; or LCN: M2 and S1J) was performed.

Histology and data exclusions. At the end of the study, animals were rendered unconscious by a rising concentration of $\mathrm{CO}_{2}$ gas for $\geq 15$ min. Brains were removed and placed in $10 \%$ $(w / v)$ buffered paraformaldehyde and shipped for histological processing (Neuroscience Associates), which involved $40 \mu \mathrm{m}$ coronal sectioning of implanted regions and staining with thionin for Nissl bodies. Upon return, microscopic assessment confirmed CPE placement with reference to a standard rat brain atlas (Paxinos and Watson, 2007). Animals with inaccurate placements were excluded from subsequent analyses, except for the M2 placements that ended up in the primary motor cortex (M1) as this forms part of the same network as seen in rsfMRI (Schwarz et al., 2013b).

Thirty-six animals began the study but three lost their implants and were culled before the end of the experiment. Animals with poor signal quality or signal losses on any of their four channels were also excluded from further analysis before data processing. After histology and signal quality exclusions, 19 animals were included in the final analysis.

\section{Results}

CPEs were implanted in pairs of nodes in DMN (PRL and RSC, corresponding to major prefrontal and posterior cingulate nodes in human DMN), and LCN (M2 and S1J; Fig. 1a,b). These pairings allowed functional connectivity to be assessed within both networks (DMN, PRL-RSC; LCN, M2-S1J), and also provided four combinations of between-network correlation (PRL-M2, PRL-S1J, M2-RSC, S1JRSC), as shown in Figure $1 c$. Recording $\mathrm{O}_{2}$ signals from animals in a state of unscheduled, spontaneous behavior revealed significant differences in correlations between node pairs $\left(F_{(2.6,47.2)}=22.6, p<0.001\right)$. Filtering the regional $\mathrm{O}_{2}$ signals $(0.01-0.1$ $\mathrm{Hz}$ ) revealed highly synchronous lowfrequency fluctuations for the two DMN nodes (PRL and RSC), but not for a between-network pair (RSC and M2) for a 
5 min resting period in one representative animal (Fig. 1d). Mean functional connectivity $(r)$ was significantly greater within the two established intrinsic network pairs compared with the betweennetwork pairs $(p<0.001$; Fig. $1 e, f)$, in accordance with a previous report of these two networks in the rodent (Schwarz et al., 2013b).

Behavioral state modulation of $\mathrm{O}_{2}$ correlations was assessed using an alternating 15 min block design (Fig. 2a), where animals were cued during task blocks to nose poke for food reward under a variableinterval $30 \mathrm{~s}$ schedule, while during rest blocks no rewards were available and behavior was not schedule controlled. Once acquired, behavioral distinction between blocks was clearly evident (Fig. 2b) with rats being significantly more active during task compared with rest blocks (nose pokes: $F_{(1,18)}=338.9, p<0.001$; movement: $\left.F_{(1,18)}=101.8, p<0.001\right)$. In terms of functional connectivity (Fig. $2 c$ ), a stable level of high correlation was evident within the LCN pairing regardless of block type $\left(F_{(1,18)}=3.2\right.$, not significant). In contrast, connectivity within the DMN pairing significantly decreased during task blocks compared with rest blocks $\left(F_{(1,18)}\right.$ $=25.4, p<0.001)$. After the blocked task was stably acquired, the rats then underwent extinction by withholding reward. Upon initiation of extinction, taskinduced behaviors decreased (Fig. 2b, right panel) yet still displayed a block effect where rats remained more active during task compared with rest blocks (nose pokes: $F_{(1,18)}=64.4, p<0.001$; movement: $\left.F_{(1,16)}=33.2, p<0.001\right)$ despite the fact that reward was no longer available. In this state, task/rest block differences in functional connectivity were lost within the DMN pair $\left(F_{(1,18)}=2.1, p=\right.$ 0.17; Fig. $2 c$, left panel), while LCN correlations remained consistently high (Fig. $2 c$, right panel). The heat maps in Figure $2 d$ illustrate connectivity profiles across network pairs at higher temporal and frequency resolutions, where reductions in DMN connectivity were task-dependent during acquisition, and lost upon extinction. In addition to nonrewarded extinction, the two other control conditions (All-Task and All-Rest) were also performed in a counterbalanced order followed by reinstatement to further probe the task-induced modulation of network functional connectivity. Figure $3 a$ shows that in all three control conditions, the task-induced reduction in DMN connectivity seen on the acquired day was lost (All-Rest, $F_{(1,18)}=2.86, p=0.11$; All-Task, $F_{(1,18)}=$ $3.51, p=0.07)$, but returned on the first day of reinstatement of the block design $\left(F_{(1,18)}=19.1, p<0.001\right)$. Connectivity within the lateral cortical network was not significantly modulated by task in any of the control conditions (all $F_{(1,18)}<1.65, p>0.22$; Fig. 3b). 19.
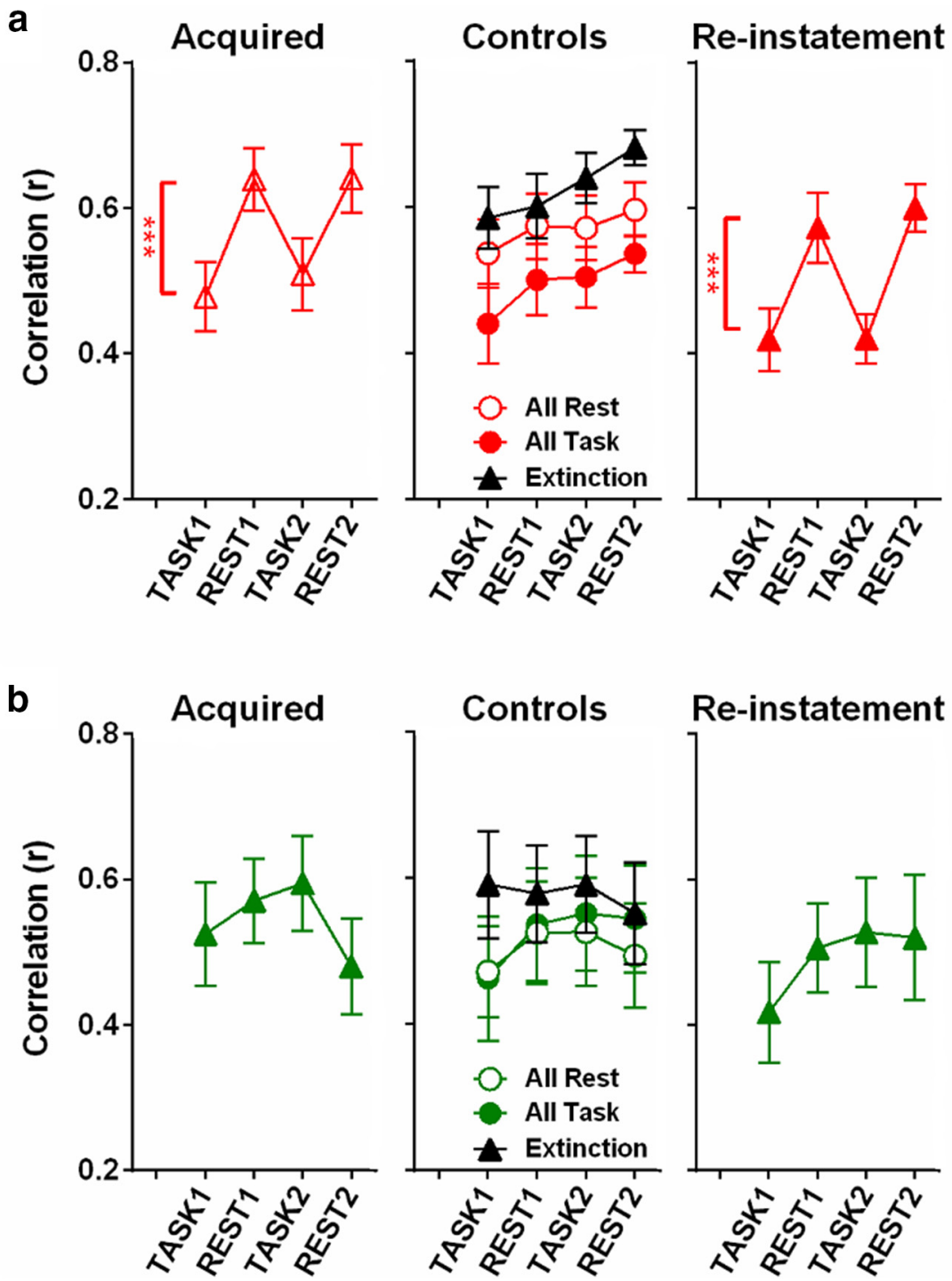

Figure 3. The effect of three control conditions on functional connectivity in the DMN and LCN. All Rest consisted of a 60 min block of unscheduled, spontaneous behavior. All Task involved a single $60 \mathrm{~min}$ block of scheduled and cued instrumental responding. Extinction consisted of the standard 15 min cued but unrewarded blocks. Reinstatement illustrates the first day when the animals were returned to the original block design. $\boldsymbol{a}$, Broadband correlations of DMN activity (PRL-RSC pair) on acquired, control, days $(p<0.001)$, mean $r \pm$ SEM, $n=19 . \boldsymbol{b}$, Broadband correlations of LCN activity (M2-S1J pair) on acquired day, control and reinstatement days per block. No significant task versus rest differences were found on any day $(p>0.05), \operatorname{mean} r \pm \mathrm{SEM}, n=$

In contrast to functional connectivity measurements, the absolute amplitude of regional $\mathrm{O}_{2}$ signals showed a clear taskinduced increase in all regions (Fig. $4 a$, mean response; Fig. $4 c$, raw $\mathrm{O}_{2}$ levels from one representative animal), regardless of their intrinsic network association. Since the regional signals did not decay back to baseline levels during rest blocks, changes for each block were normalized to previous block levels and summarized by a mean AUC measure in Figure $4 b$. Again, a task-induced increase in signal amplitude was evident in all regions (Block state, $F_{(1,18)}=85.5, p<0.001$; Region $\times$ Block state, $F_{(3,54)}=$ 10.8, $p<0.001)$.

\section{Discussion}

Using $\mathrm{O}_{2}$ amperometry functional connectivity measures as a homolog of rsfMRI, intrinsic network correlations comparable to those identified in the anesthetized rodent by rsfMRI were de- 
a

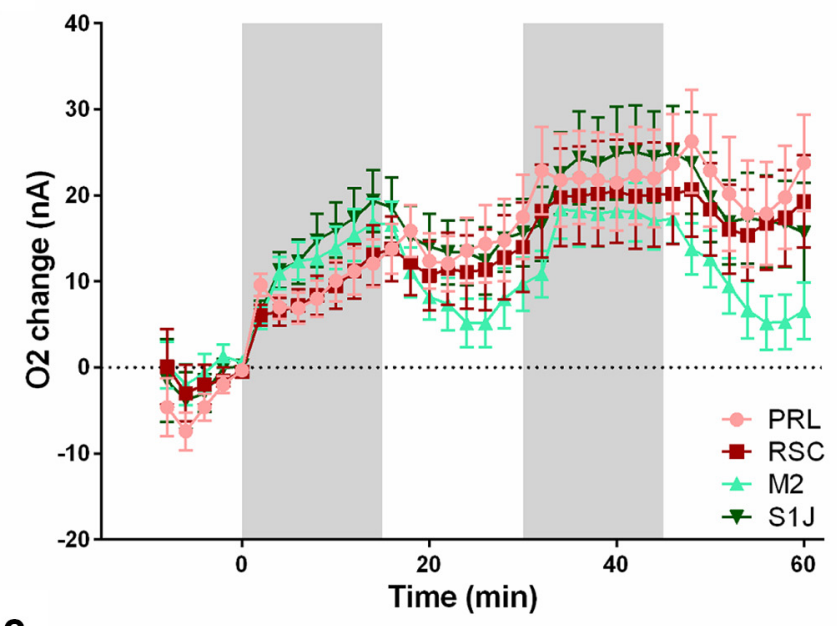

C

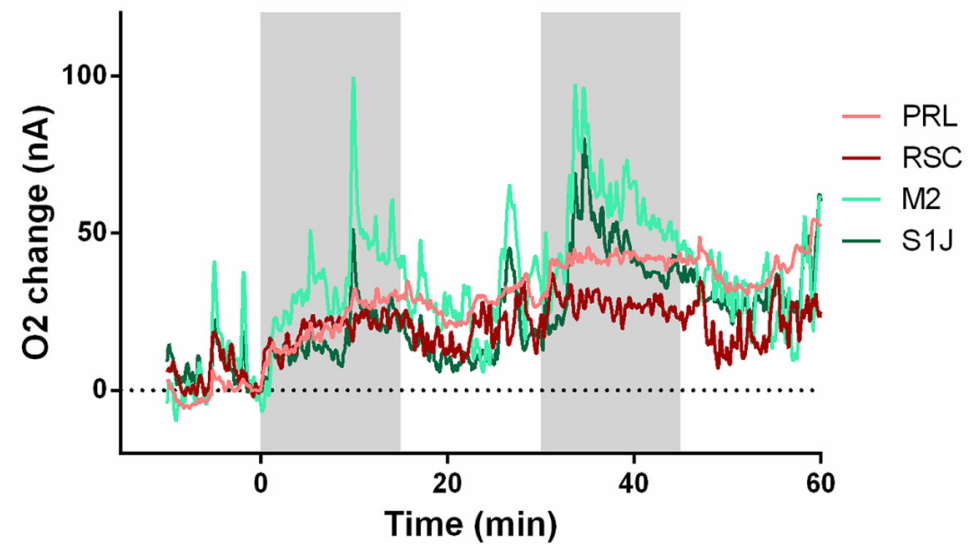

b

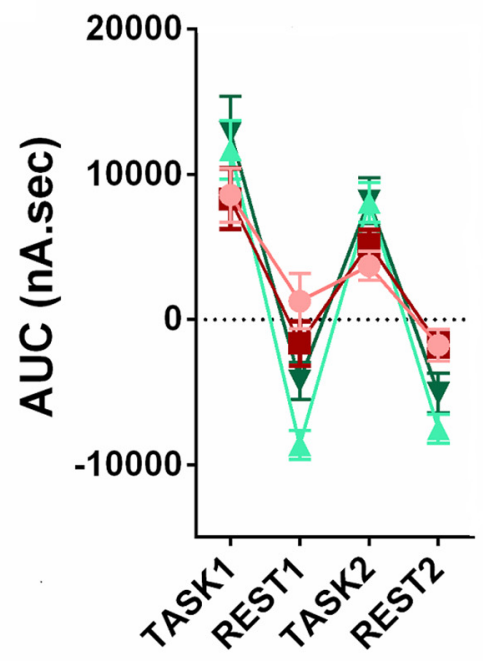

Figure 4. Task-induced modulation of regional $\mathrm{O}_{2}$ response. $\boldsymbol{a}$, Average time course of absolute regional $\mathrm{O}_{2}$ responses (task blocks indicated in gray) from all animals. Values are mean \pm SEM,

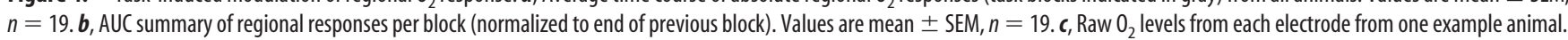

tected in freely moving rats. Greater levels of connectivity were measured between node pairs within two discrete networks, the DMN and the LCN, in contrast to significantly lower crossnetwork connectivity strength. Moreover, engagement in instrumental action elicited robust decreases in functional connectivity selectively within the DMN, but not the LCN. These results demonstrate that intrinsic network connectivity can be modulated by task engagement in the behaving rat, opening the way for exploration of the effects of other cognitive tasks and increasing the opportunities to translate rodent functional connectivity studies to human rsfMRI. A lack of correlation (or anticorrelation) between task-positive and task-negative networks such as the DMN has been well documented in fMRI recordings in humans (Fox et al., 2005; Fransson, 2006), rodents (Schwarz et al., 2013b; Sforazzini et al., 2014), and also in direct neuronal recordings of local field potentials from implanted electrodes in animals (Popa et al., 2009). In line with these observations, the current study demonstrates low-frequency amperometric $\mathrm{O}_{2}$ recordings in freely moving rats also show a low level of correlation between regions known to be part of different intrinsic networks, highlighting the translational potential of in vivo $\mathrm{O}_{2}$ amperometry technique.

Despite a stable level of high baseline correlation within both networks, connectivity only within the DMN pairing significantly decreased during task blocks compared with rest blocks. Thus, functional connectivity between the pair of nodes within rat $\mathrm{DMN}$ was sensitive to behavioral state and became relatively more disengaged during scheduled task performance, in line with some human rsfMRI findings (Fransson, 2006). The present $\mathrm{O}_{2}$ data are also consistent with a study comparing the functional connectivity of BOLD, perfusion, and CMRO2 (cerebral metabolic rate of $\mathrm{O}_{2}$ consumption) signals in healthy human volunteers both at rest and undergoing a visual task (Wu et al., 2009). They observed that connectivity of the CMRO2 signal was similar to BOLD connectivity, with both showing low connectivity strength within the DMN during a visual stimulation task compared with the resting state. Note that there are also reports of increased DMN functional connectivity during task performance (Li et al., 2012), highlighting the mixed findings in the human imaging literature, which may be due to the different tasks and brain regions considered. Upon initiation of Extinction and AllRest and All-Task control conditions, the task/rest block differences in functional connectivity were lost within the DMN pair, but returned immediately upon reinstatement of the block design. This suggests that the DMN connectivity changes are not a straightforward measurement of movement, stimulus perception, or temporal artifact, and are likely driven by another aspect of task engagement.

Regional amplitude responses showed a clear task-induced increase in tissue $\mathrm{O}_{2}$ in all four regions regardless of their location and assumed network role. This contrasts with commonly reported task-induced regional decreases (or deactivations) in hu- 
man DMN (Raichle et al., 2001; Anticevic et al., 2012). However, a recent human imaging study reported activation of both DMN regions and task-positive regions when subjects undergo preparatory phases of a working memory task (Koshino et al., 2014). The extent of DMN changes may therefore depend on the nature of the task and associated cognitive load. The rat resting and task state in the current study may be fundamentally different from the equivalent human states, and thus may not result in the same $\mathrm{O}_{2} /$ BOLD deactivations as the regional human BOLD response. This is difficult to test since behavior cannot be probed in rodent fMRI studies. Therefore it is not yet possible to determine whether the results reflect a species difference over a technical difference. Also, it cannot be ruled out that movement/activity of animals may be influencing the regional response, particularly since an increase was seen in all regions during the task. Previous studies in this laboratory have not shown any influence of head movement on the $\mathrm{O}_{2}$ signal per se, but regional $\mathrm{O}_{2}$ changes and overall activity of the animal have yet to be fully dissociated.

In contrast to the DMN nodes, where both amplitude and connectivity measures were sensitive to task status, LCN nodes showed a task-related regional response but functional connectivity was not significantly affected by task performance, suggesting that regional amplitude and correlation measures can be differentially modulated by behavioral state and hence are functionally dissociable. Despite remaining questions over the mechanistic equivalence of the DMN regional response to commonly reported human deactivation responses, connectivity measures exhibit the properties of network-specific and task-induced modulation, both of which qualitatively mirror human DMN behavior.

The degree to which $\mathrm{O}_{2}$ amperometric signals can be considered as a proxy for BOLD signal changes measured in fMRI studies is a key factor in the translatability of this approach. Evidence to date reveals strong empirical parallels between observations using $\mathrm{O}_{2}$ amperometry and findings from rat and human fMRI. It has been shown that $\mathrm{O}_{2}$ signal changes evoked by hyperoxia and hypoxia closely parallel BOLD signal changes in the rat brain (Lowry et al., 2010), and that localized $\mathrm{O}_{2}$ increases in the nucleus accumbens associated with reward anticipation are strikingly consistent with BOLD signal changes observed in response to reward anticipation conditions in human fMRI (Francois et al., 2012). Finally, consistent pharmacological alterations in functional connectivity as measured by $\mathrm{O}_{2}$ amperometry and by rsfMRI have been reported, using acute ketamine as the pharmacological probe (Gass et al., 2014; Li et al., 2014).

However, several differences and limitations to rat and human fMRI are nevertheless relevant. First, a resting-state comparable to that defined in human imaging work may not exist in a freely moving rodent, even with the most controlled conditions (quiet, stimulus free). While clearly an evolution over rat imaging studies, where animals are anesthetized and not at rest by human study definition, the rest state in the present study reflects a dynamic process of habituation of spontaneous, unscheduled behavior, which produced a distinction to that of the task state. It may therefore be very important for future translational work to more accurately align and control what is meant by the rest state in both rats and humans. A second limitation of the $\mathrm{O}_{2}$ technique is the currently limited number of brain regions that can practically be recorded from (four), compared with the whole-brain measurement possible with fMRI. In amperometric $\mathrm{O}_{2}$ functional connectivity analyses, sampled brain regions have to be determined a priori, typically meaning that a complete network cannot be elucidated from a single study as the maximum num- ber of node-to-node connectivity pairs is currently limited to six. However, by selecting key regions representative of known networks as informed by imaging studies, specific hypotheses regarding the modulation of brain activity by behavior can be tested. This important advantage over rodent fMRI, and the close parallels observed between $\mathrm{O}_{2}$ and BOLD responses over a range of paradigms and conditions, provide confidence that recording from freely moving rodents undergoing behavioral tasks using this approach will help bridge the imaging gap in translational behavioral responses.

Future work should focus on gaining a deeper understanding of cross-species homology of such intrinsic network activity during controlled, differential expression of specific behaviors. The instrumental response schedule used in the current study was a very simple task that has limited potential to measure cognitive load. To enhance understanding of the translational correspondence of task-induced intrinsic connectivity patterns, a richer array of tasks, back-translated from human studies, needs to be performed in rodents to probe the extent to which the behavior of the rodent "DMN" and other networks parallel the human homologs. Since many different brain regions are known to be associated with the DMN in both humans and rodents, further studies are also needed to probe other regions in the rodent to determine whether task-related reductions in connectivity are consistent across all nodes of the DMN.

In summary, engagement in instrumental action elicited robust decreases in functional connectivity selectively within node pairs of the DMN, but not the $\mathrm{LCN}$, in the awake, behaving rat. This task-associated modulation, abolished during task extinction, was not caused by elevated movement per se and reappeared upon task reinstatement, confirming both behavioral dependency and network-specificity of this effect. The ability to assess in vivo amperometric $\mathrm{O}_{2}$ signal proxies of $\mathrm{BOLD}$-like functional connectivity measures in awake, freely moving animals greatly enlarges the range of behavioral paradigms that can be backtranslated from human studies, potentially taking preclinical intrinsic network research beyond the "resting state."

\section{References}

Anticevic A, Cole MW, Murray JD, Corlett PR, Wang XJ, Krystal JH (2012) The role of default network deactivation in cognition and disease. Trends Cogn Sci 16:584-592. CrossRef Medline

Becerra L, Pendse G, Chang PC, Bishop J, Borsook D (2011) Robust reproducible resting state networks in the awake rodent brain. PLoS One 6:e25701. CrossRef Medline

Beckmann CF, DeLuca M, Devlin JT, Smith SM (2005) Investigations into resting-state connectivity using independent component analysis. Philos Trans R Soc Lond B Biol Sci 360:1001-1013. CrossRef Medline

Bolger FB, McHugh SB, Bennett R, Li J, Ishiwari K, Francois J, Conway MW, Gilmour G, Bannerman DM, Fillenz M, Tricklebank M, Lowry JP (2011) Characterisation of carbon paste electrodes for real-time amperometric monitoring of brain tissue oxygen. J Neurosci Methods 195:135-142. CrossRef Medline

Esslinger C, Walter H, Kirsch P, Erk S, Schnell K, Arnold C, Haddad L, Mier D, Opitz von Boberfeld C, Raab K, Witt SH, Rietschel M, Cichon S, Meyer-Lindenberg A (2009) Neural mechanisms of a genome-wide supported psychosis variant. Science 324:605. CrossRef Medline

Fox MD, Greicius M (2010) Clinical applications of resting state functional connectivity. Front Syst Neurosci 4:19. CrossRef Medline

Fox MD, Snyder AZ, Vincent JL, Corbetta M, Van Essen DC, Raichle ME (2005) The human brain is intrinsically organized into dynamic, anticorrelated functional networks. Proc Natl Acad Sci U S A 102:9673-9678. CrossRef Medline

Francois J, Conway MW, Lowry JP, Tricklebank MD, Gilmour G (2012) Changes in reward-related signals in the rat nucleus accumbens measured by in vivo oxygen amperometry are consistent with fMRI BOLD responses in man. Neuroimage 60:2169-2181. CrossRef Medline 
Fransson P (2006) How default is the default mode of brain function? Further evidence from intrinsic BOLD signal fluctuations. Neuropsychologia 44:2836-2845. CrossRef Medline

Gass N, Schwarz AJ, Sartorius A, Schenker E, Risterucci C, Spedding M, Zheng L, Meyer-Lindenberg A, Weber-Fahr W (2014) Sub-anesthetic ketamine modulates intrinsic BOLD connectivity within the hippocampal-prefrontal circuit in the rat. Neuropsychopharmacology 39:895-906. CrossRef Medline

Koshino H, Minamoto T, Yaoi K, Osaka M, Osaka N (2014) Coactivation of the default mode network regions and working memory network regions during task preparation. Sci Rep 4:5954. CrossRef Medline

Li B, Wang X, Yao S, Hu D, Friston K (2012) Task-dependent modulation of effective connectivity within the default mode network. Front Psychol 3:206. CrossRef Medline

Li J, Ishiwari K, Conway MW, Francois J, Huxter J, Lowry JP, Schwarz AJ, Tricklebank M, Gilmour G (2014) Dissociable effects of antipsychotics on ketamine-induced changes in regional oxygenation and inter-regional coherence of low frequency oxygen fluctuations in the rat. Neuropsychopharmacology 39:1635-1644. CrossRef Medline

Lowry JP, Boutelle MG, Fillenz M (1997) Measurement of brain tissue oxygen at a carbon paste electrode can serve as an index of increases in regional cerebral blood flow. J Neurosci Methods 71:177-182. CrossRef Medline

Lowry JP, Griffin K, McHugh SB, Lowe AS, Tricklebank M, Sibson NR (2010) Real-time electrochemical monitoring of brain tissue oxygen: a surrogate for functional magnetic resonance imaging in rodents. Neuroimage 52:549-555. CrossRef Medline

Lu H, Zou Q, Gu H, Raichle ME, Stein EA, Yang Y (2012) Rat brains also have a default mode network. Proc Natl Acad Sci U S A 109:3979-3984. CrossRef Medline

McHugh SB, Marques-Smith A, Li J, Rawlins JN, Lowry J, Conway M, Gilmour G, Tricklebank M, Bannerman DM (2013) Hemodynamic responses in amygdala and hippocampus distinguish between aversive and neutral cues during Pavlovian fear conditioning in behaving rats. Eur J Neurosci 37:498-507. CrossRef Medline
O’Neill RD, Grünewald RA, Fillenz M, Albery WJ (1982) Linear sweep voltammetry with carbon paste electrodes in the rat striatum. Neuroscience 7:1945-1954. CrossRef Medline

Paxinos G, Watson C (2007) The rat brain in stereotaxic coordinates. 6th ed. San Diego: Elsevier Academic.

Popa D, Popescu AT, Paré D (2009) Contrasting activity profile of two distributed cortical networks as a function of attentional demands. J Neurosci 29:1191-1201. CrossRef Medline

Raichle ME, MacLeod AM, Snyder AZ, Powers WJ, Gusnard DA, Shulman GL (2001) A default mode of brain function. Proc Natl Acad Sci U S A 98:676-682. CrossRef Medline

Schwarz AJ, Gass N, Sartorius A, Zheng L, Spedding M, Schenker E, Risterucci C, Meyer-Lindenberg A, Weber-Fahr W (2013a) The low-frequency blood oxygenation level-dependent functional connectivity signature of the hippocampal-prefrontal network in the rat brain. Neuroscience 228: 243-258. CrossRef Medline

Schwarz AJ, Gass N, Sartorius A, Risterucci C, Spedding M, Schenker E, Meyer-Lindenberg A, Weber-Fahr W (2013b) Anti-correlated cortical networks of intrinsic connectivity in the rat brain. Brain Connect 3:503511. CrossRef Medline

Sforazzini F, Schwarz AJ, Galbusera A, Bifone A, Gozzi A (2014) Distributed BOLD and CBV-weighted resting-state networks in the mouse brain. Neuroimage 87:403-415. CrossRef Medline

Shirer WR, Ryali S, Rykhlevskaia E, Menon V, Greicius MD (2012) Decoding subject-driven cognitive states with whole-brain connectivity patterns. Cereb Cortex 22:158-165. CrossRef Medline

Smith SM, Fox PT, Miller KL, Glahn DC, Fox PM, Mackay CE, Filippini N, Watkins KE, Toro R, Laird AR, Beckmann CF (2009) Correspondence of the brain's functional architecture during activation and rest. Proc Natl Acad Sci U S A 106:13040-13045. CrossRef Medline

Wu CW, Gu H, Lu H, Stein EA, Chen JH, Yang Y (2009) Mapping functional connectivity based on synchronized CMRO2 fluctuations during the resting state. Neuroimage 45:694-701. CrossRef Medline 